\title{
Bringing Health Care Home: A Pilot Project to Improve Access to Primary Health Care Using Mobile Nurse Practitioner Services
}

\author{
By Donna Clare*
}

\begin{abstract}
In the last decade in Canada, goods and services delivered to one's doorstep has become commonplace. In most major Canadian cities, anyone can easily arrange for food and drug delivery, massage and physical therapy, dental care and even dog grooming in one's home. At the same time, primary health care provision in the community remains almost exclusively delivered in the clinic or facility setting, and within those services, health promotion and selfcare education is notably sparse. One innovative organization in Calgary, Canada, has piloted a program to provide health care in clients' homes. Services include comprehensive physicals and screening, prescribing, phlebotomy and specimen collection, wound care, referrals, counselling, and health promotion and self-care education for clients of all ages. Nurse Practitioners were chosen to provide these services based on their ability to provide the full spectrum of these health services. In addition to home visits, the Nurse Practitioners provide phone, video and email consultations depending on the situation, and access is 24/7. Pilot analytics include: Costs of the service, Cost savings to the public health care system, Client satisfaction, Nurse Practitioner satisfaction, Integration of health promotion and self-care, Comfort and use of multimedia (video, phone, email) by both clients and NPs, Challenges, Success stories.
\end{abstract}

Keywords: Health Promotion, Innovation, Mobile Health Care, Nurse Practitioner, Primary Care Delivery

\section{Introduction}

Private healthcare organizations are responsive to trends in the global marketplace and are often early adopters of technology and other innovations to meet the needs of their clients (Lister et al. 2017, Omachonu and Einspruch 2010). The Canadian public's growing demand for convenience and personalized care means that a myriad of services, traditionally available in storefronts, offices and clinics, are now provided right to the door or in one's own home. Physical therapists, dental hygienists, hair stylists, dog groomers and veterinarians, and a plethora of others, have risen to the challenge and now provide mobile services.

Busy, technologically-savvy Canadians have come to expect services that make their lives easier (Musser 2019, Government of Canada 2012). Boomers (those born between 1944 and 1964) are advocating for home-based services that support aging-in-place for their elderly parents (CHSRF 2011), and Millennials (those born between 1980 and 1994) want easy access to health care providers using text messaging and video conferencing, as well as information that is technology-based and yet, personal (Sandomirsky 2018, Walker 2018). Primary care, defined as the initial interface between patient and health care provider, usually a physician's clinic in the community (Canadian Nurses Association

\footnotetext{
*Academic Coordinator, Athabasca University, Canada.
} 
2013), has not kept pace with public demand for convenience, greater accessibility and patient-centre care (Health Canada 2005). This paper describes a pilot project that addresses these demands by re-imagining the medical house call.

\section{Background}

There are economic benefits to home-based health care. The growing population of seniors in Canada is necessitating a call for innovation in primary care delivery if only to reduce expensive hospital and emergency room use downstream (Herritt 2015, Leff et al. 2015). There are an estimated 100,000 homebound individuals in Canada, for whom access to primary care is a challenge (Stall et al. 2013); and a recent study, by Heale et al. (2018), showed that chronic disease management often suffers when patients with limited resources are obliged to travel to a clinic for care and there are no alternatives. Homebound individuals and those with chronic diseases are more likely be hospitalized and use the emergency room (Densai et al. 2008, Norman et al. 2018, Woolf et al. 2015); whereas, research demonstrates that house calls result in reduce use of both the emergency rooms and hospitals (Chapman et al. 2018, Schuchman et al. 2018).

Providing primary care in the home used to be commonplace in Canada, but by the 1940 's, family physician house calls began to wane, and by the late 1960's very few physicians were going to see patients in their homes (Herritt 2015). Today, physicians are still reluctant to provide house calls (Smith and Dhalla 2011, Meyers 2015) however, recent advances in telecommunications technology, especially secure video and chat services, together with the addition of nurse practitioners to the mix of available primary health care providers, makes innovation in the sector possible. In fact, in the USA, more nurse practitioners are providing medical house calls than any other provider including physicians (Yao et al. 2016). Nurses have a long tradition of providing care to people wherever they are, be it the battlefield, the home or school. The Canadian Nurses Association (2013) predicts that nurse practitioners and registered nurses will instrumental in providing comprehensive home-based health care in the coming years.

In response to these trends, one Calgary-based organization that provides private health care, fitness and medical-esthetic services to its clients, began a pilot project to offer medical house calls. The clients of the organization expressed a desire for improved access to primary care, preferable in the home or office setting, more health promotion counseling, and greater integration of illness prevention care beyond screening. The 8 in-house physicians, who at the time offered comprehensive health care services in the organization's clinic setting, preferred not to expand their role in this way, and so the decision was made to pilot a program using Nurse Practitioners (NPs) to provide mobile, 24/7, primary care services. 


\section{Aims of the Pilot Project}

The project aims to:

- Evaluate the feasibility of providing house calls and telehealth services using NPs.

- Inform a business decision to proceed (or not) with upscaling the service within the organization and expanding it nation-wide.

Taking a bigger view, the company hopes that this pilot, and any future service innovations proceeding from its outcomes, may contribute innovations transferable to primary care within Canada's publicly-funded healthcare system. The company also believes that by providing high-quality primary care in the home, there will be a reduction in the use of hospitals and emergency rooms, thereby saving the healthcare system money.

What follows is a report on the structure of the pilot program, methods of evaluation, key findings, and lessons learned.

\section{Framework}

A 6-month pilot (July to December) was proposed, enrolling up to 250 people of all ages. The pilot project was funded by the organization's holding company, and supported by pre-existing resources within the organization, e.g., finance, information technology, operations, graphic design, and communications.

\section{Service Model}

Nurse Practitioners were chosen to provide these services based on their ability to provide the full spectrum of primary care services including assessments, diagnosis, ordering diagnostics and making referrals, as well as prescribing and phlebotomy.

Services offered in this pilot included evidence-based comprehensive physicals and screening for disease, treatment of acute and chronic conditions, phlebotomy and specimen collection, prescribing, referrals to specialist, as well as health promotion counseling, integration of illness prevention strategies, and selfcare education for clients of all ages, in the home or office environment as desired by the client.

Each client's first visit was conducted in the home and included a full health history interview. Afterwards, clients were free to use the telehealth services (video, email or phone) as appropriate. The NPs were available for house calls on weekdays from 10 am to $7 \mathrm{pm}$ and rotated on-call duty on the off hours (for phone or video contact) so that access was $24 / 7$.

The NPs shared the care of the entire client roster of pilot enrollees. Each NP was enrolled into the electronic medical record which was also used by the physician group within the organization. A designated phone number for the 
mobile service was forwarded to the NP's personal cellular phones. NPs were provided with a small laptop and a portable printer-fax machine as well as two carrying cases of equipment including a portable weigh scale, and a centrifuge that would work in their car. NPs delivered lab the specimens directly to the appropriate labs. NPs were hired as independent contractors and were paid an hourly wage, plus mileage. They provided their own vehicle and professional liability insurance.

\section{NP Qualifications}

Initially, two NPs were hired: One NP with 10 years' experience working in primary care, and a new graduate who had experience in home care nursing. The former served as the NP-Lead and had strong skills in patient-centered care and NP integration processes; the new NP was able to advise on issues regarding safety strategies for going into patient's homes. A third NP was added to the team two months into the pilot when the demand for services increased. This last NP was also an experienced community NP with 7 years' experience. All NPs graduated from the same University so were aware of each other's strengths and knowledge base.

\section{Client Recruitment}

The company chose to invite three types of participants, all of which were identified by the company as potential users of such a service:

1. Current clients who subscribe to the company's current concierge health care and aesthetic services;

2. Clients identified by the company physicians who might benefit or want house calls because of chronic disease or mobility issues. This accounted for only 20 of the total number recruited, and,

3. Persons in the community who might be interested in such a service but who are not clients of this company's nor any other company's concierge health care services. Participants in this last group were recruited via snowball sampling initiated through the friends and families of the company's executive team, thereby limiting communication of this pilot project to the company's competitors.

The services were offered free of charge to the enrollees for the duration of the pilot. None of the services, including the telehealth services, continued once the pilot was completed.

\section{NP Integration Process}

Nurse practitioners are still relatively new in Canada and most organizations require advice on how to integrate NPs into an existing practice milieu or new service (Bryant-Lukosius and Dicenso 2004). Consequently, a NP leader was 
hired initially to advise both management and in-house physicians on NP scope of practice, develop an orientation program, draft policies and procedures, and to organize equipment relevant to NP practice. Contractual details, being somewhat unique from physician practice, were developed in consultation with the Canadian Nurses Protective Society.

The NPs met with the physicians, the operations team and the organization's executive team several times before start-up. In keeping with Bryant-Lukosius and DiCenso's (2004) PEPPA framework for successful NP integration, expectations were aligned, knowledge of the NP scope of practice and role in health care provision was shared, and how NPs would communicate and interact with other parts of the organization was discussed. The main concerns expressed by the physicians were in regard to communication, and legal liability in the case of shared care.

In response to physician concerns, the NP lead prepared a document offering a literature review and discussion on collaborative care. Then, together with the physicians and to mutual satisfaction, a policy was drafted outlining lines of communication and delineating mutual responsibilities according to the professional obligations of each party.

\section{Method}

At the end of the six months, enrollment ceased, and data collection commenced, however, the pilot ran for a total of 9 months (July to March), giving those who enrolled in the fourth and fifth months, the opportunity to continue to use the service and form an opinion for evaluation purposes. Clients were interviewed and surveyed after they had been enrolled in the service for a least 5 months. Contact with clients and all services ceased once the pilot evaluation was complete; for some, this meant that they continued to have access to the services after providing feedback. The NPs were unaware of who had participated in the evaluation process and who had not.

A practical paradigm using both qualitative and quantitative methodologies was employed. Anonymized client profiles were compiled as congregate data, including what services were provided and the medium used for the encounter (e.g., phone, video, email or home visit). Enrollees were surveyed using multiple choice, Likert scales and short answer. Focus groups were held, and many clients were interviewed by phone by an employee of the organization not involved in operations or service provision. The NPs were surveyed using the Misener Nurse Practitioner Job Satisfaction Scale (Martin-Misener 2000) and short interview. A workflow audit was conducted by the lead NP.

Unfortunately, the company chose not to submit a formal ethics application citing time constraints, however, no breaches of ethical research standards were noted by this author. Specifically, confidentiality and privacy of patient information was maintained and the privacy officer within the company provided oversight. 
Vol. 6, No. 1 Clare: Bringing Health Care Home: A Pilot Project to Improve Access...

\section{Results}

\section{Client Demographics}

A total of 254 people enrolled in the pilot (Table 1). It was anticipated that the elderly, because of limitation in mobility, weather conditions and higher rates of chronic disease would be the heaviest users of the service, but the results showed that dual-income couples with children utilized the service most often.

Patient quote (paraphrased) "I was so grateful that they could come to my home. Our family was going through a lot at the time and it was so much easier than having to get to doctor's appointments, too."

Table 1. Encounters by Age Group (Not Including the Initial Intake Visit)

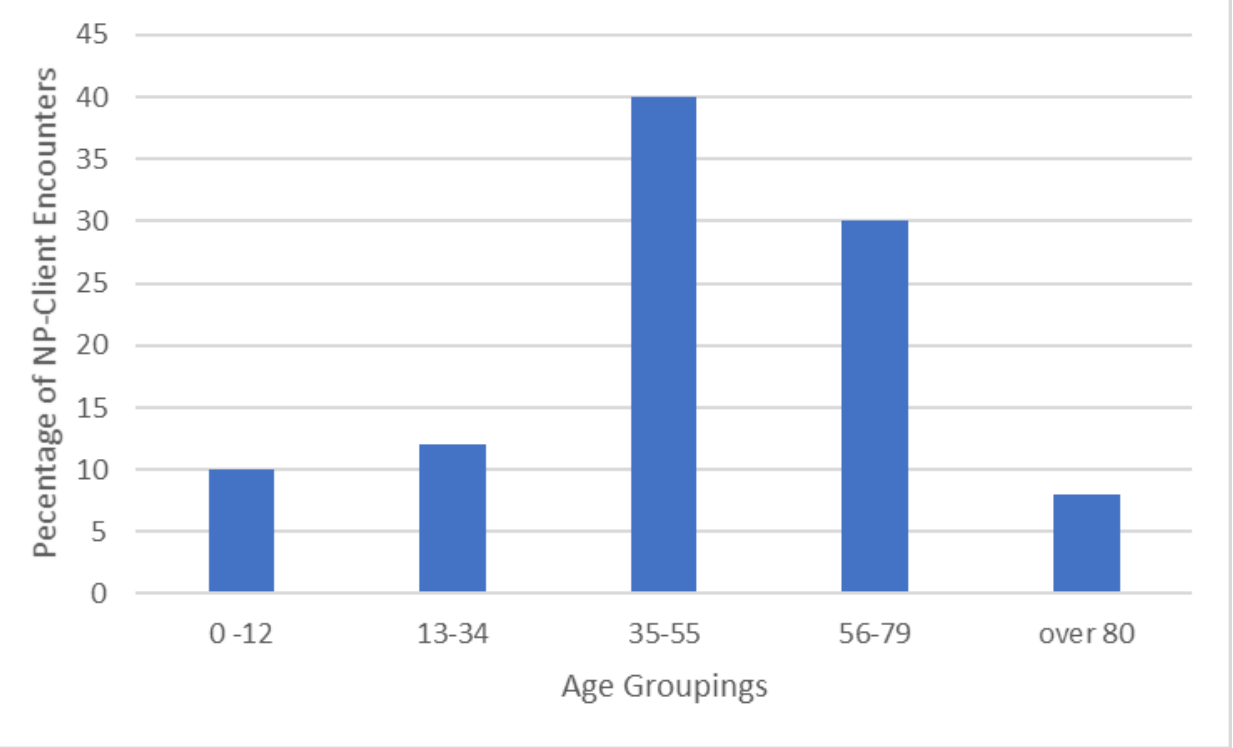

By gender: $51 \%$ males/49\% female (includes children).

\section{Health Conditions}

The most common conditions seen by the NPs match those common acute and chronic diseases seen in traditional primary care clinic settings. The most common procedures performed by the NPs were ear lavage, PAP tests, and phlebotomy.

The most frequent reason for a client encounter (any medium):

- Periodic Health Examination.

- Stress-related disorders.

- Skin problems.

- Urinary tract infections.

- Upper respiratory tract infections. 
- Prescription refills.

- Lab collection.

- Follow-up care or health counseling.

\section{Most Common Health Promotion and Illness Prevention Interventions}

Health promotion and illness prevention strategies were offered at almost all client encounters (any media). A workflow record compiled by the NPs, revealed that both medical (e.g., diagnosis, labs, prescriptions) and nursing strategies (e.g., self-care, immunizations, health promotion) were offered depending on the situation. Health education was integral to each encounter and most often included information about a particular health condition, self-care or medication information, or dispelling myths and misconceptions.

Health Promotion and illness prevention strategies most often included in client visits:

- Vitamin D supplementation.

- Vaccinations, including influenza and travel immunizations.

- Screening procedures.

- Self-care advice.

- Parenting advice.

- Nutrition and special diets.

- Smoking Cessation counseling.

- Probiotic recommendations when antibiotics are prescribed.

\section{Media Evaluation}

All initial client encounters occurred in the client's home. Both the NPs and the clients enjoyed the home visit setting. Clients reported feeling more relaxed and the NPs reported appreciating the first-hand living-context of the client which they said directly affected their assessments and health care recommendations. Children were examined with a parent present.

One NP stated, "Watching (the client's) constant movement during the visit: going from kitchen to dining table, to the backdoor, to the pile on the floor and so on, told me so much about her underlying condition. My diagnosis and treatment plan was greatly informed by watching (the client) in her natural setting."

A workflow audit showed that the majority of NP-client encounters after the initial home visit, were conducted by phone (43\%), and secure email $(23 \%)$; very few were video visits (4\%) presumably due to implementation of this medium late in the pilot. Secure video visits were useful for counseling, looking at rashes, and other situations where a hands-on examination was not required, or when preferred by clients. Clients preferred emails for quick questions.

Some of the NPs found the video visits more cumbersome to set up for each encounter. One NP said, "By the time I got the video set up and running, I was frazzled because I was thinking about how much emphasis the company put on great service. There was also a big push by the company to use this new secure 
video. It didn't feel very efficient to me and it was especially hard to do if I was in a car. I didn't like using the video and avoided it if I could."

\section{Client Satisfaction}

The organization's key goal for the NPs was to provide exceptional service. Client surveys revealed an overall $96 \%$ satisfaction rating for the NP service. All interviewed enrollees expressed continued interest in continuing with the services provided by NPs and $73 \%$ found that it filled a need (Table 2 ).

Table 2. Aggregate Satisfaction Measures Rated Out of 10

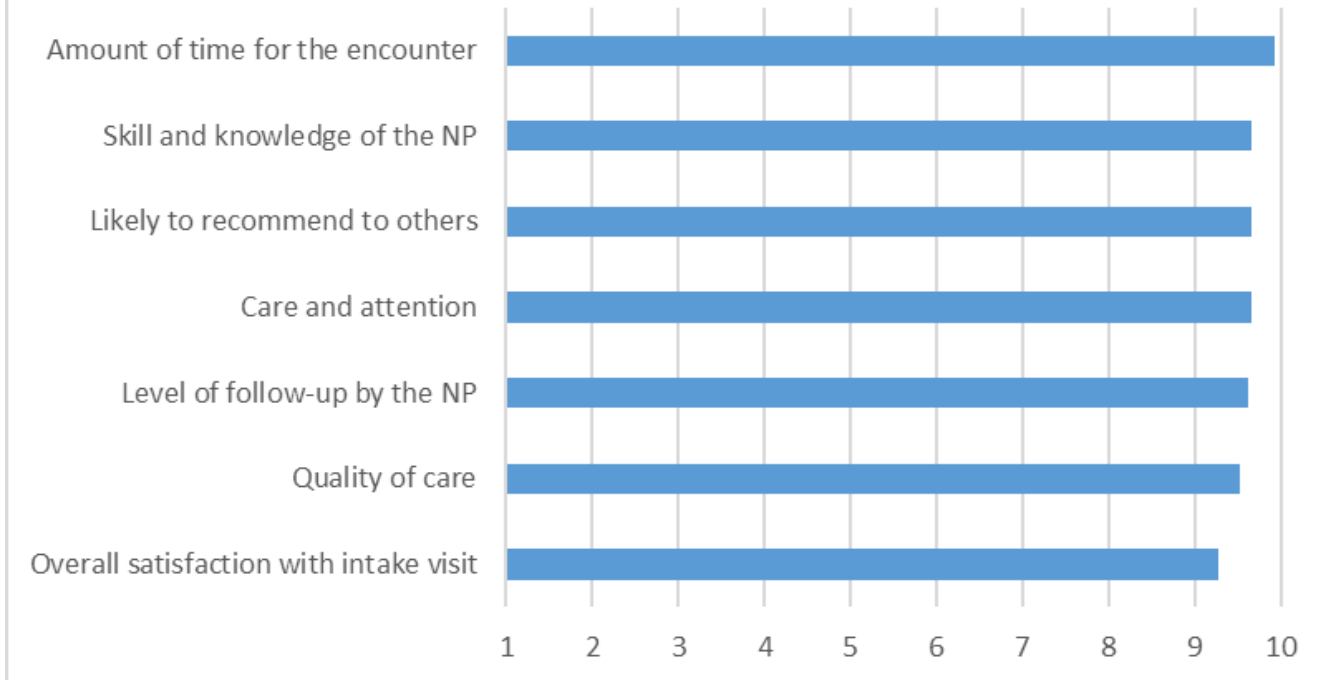

Physician Satisfaction

On reflection, the biggest challenge for both NPs and physicians was to foster the physician-NP relationships when the NPs worked off site. To this end, it was determined that more in-clinic contact would help and so the NPs were placed in the clinic to cover MD absence at the three-month mark of the pilot.

By the 6-month mark, all physicians had settled into the collaborative model and were satisfied with the care provided by the NPs and communication patterns. By the end of the pilot, an NP was hired for the on-site clinic to cover MD absences.

\section{NP Job Satisfaction}

The NPs reported that they enjoyed their role:

- The NPs enjoyed the autonomy of their role and utilizing their full scope of practice.

- The NPs were very satisfied with the patient care component of their role. They felt that had enough time to provide complete patient-centred care, 
because times allotted for each appointment was generous (1/2 to 1 hour) compared to typical primary care clinic settings (10-15 minutes).

- The NPs also enjoyed their working relationships with the physicians.

Downsides included:

- The hours of mobile work from 10 am to $7 \mathrm{pm}$ were unpopular with all of the NPs.

- Some differences of opinion amongst the NPs arose over policy e.g., whether injectables like vaccines or blood draws for children should be done in their home, or if should the home be kept a "safe place" with those painful procedures best done elsewhere (like a pharmacy, lab, public health unit).

- The mobile technology was often frustrating, meaning that charting, faxing and scanning often had to be completed after patient visits and sometimes in vehicles.

- The NPs mentioned that working in isolation with little opportunity for interaction with colleagues was less satisfying than working, say, in a clinic setting.

\section{Other Findings}

The role of the NPs evolved over the pilot. The NPs, initially, felt overwhelmed managing emails, phone calls and client visits as well as tending to faxing of referrals and prescriptions, and delivering lab specimens to lab outlets throughout the city. Administrative support was added very late in the pilot but was a great relief to the NPs.

Managing video appointments and incoming calls while mobile was a challenge. The NPs sometimes felt that the obligations to provide efficient, timely care was difficult to achieve in the context of professional obligations, especially ensuring privacy and confidentiality when conducting a video consultation in the car between scheduled home visits or talking with a client on the phone. As well, driving while using a cell phone is unlawful in Canada.

The NPs all reported that they thought the initial home visit provided an opportunity for health- history-taking and patient assessment that increased their level of comfort taking phone calls from the clients later. They felt that this was an essential component to providing safe care for their clients.

\section{Costs and Savings}

A financial review determined that the cost of the service to the organization was estimated to be $\$ 120 /$ hour CAD (\$90 USD; $€ 80$ Eur). And start-up costs ran to about $\$ 5000$ CAD (\$3750 USD; $€ 3300$ Eur) per NP. Analytic parameters included NP wages, supplies, transportation, cost of support staff, storage space and office for NPs, graphic design (handouts, forms), information technology support; electronic medical record and telephone and video service, hardware 
(computers, mobile fax/printer), business insurance, and average time of each patient encounter.

Savings to the healthcare system were hard to determine. Only one NP-client encounter, out of more than 600 care episodes, could be said to have avoided an emergency room visit for acute symptom management. None of the enrollees were admitted to hospital during the pilot, but neither was there any evidence that care provided by the NPs was instrumental in preventing admissions during this period. Long-term prevention of hospitalization cannot be determined.

\section{Unanticipated Results}

Some interesting and unanticipated patterns were observed over the course of the pilot that are worth some mention.

Some clients were late returning to their homes for a scheduled appointment, leaving the NP waiting in the winter weather outside. On the other hand, there were no missed appointments.

Approximately half of the women requesting a periodic health assessment (annual physical), were uncomfortable with having a PAP smear done in their homes. The organization did not want to accommodate those women within their physician-clinic facility, therefore, women were sometimes referred to a community women's health clinic for this procedure. NPs admitted being somewhat uncomfortable performing this procedure in homes as well. One NP stated, "It just didn't feel clinical enough for such an intimate procedure."

NPs noted that extra time was needed for in home visits because of having little control over the environment. One NP exclaimed, "Sometimes dinner preparations were in progress during the history-taking, or once a small child insisted on climbing over the parent who was being examined, or the dog put its head in the way of the phlebotomy...It can be difficult to stay on track!"

Working within a business model rather than a health care model was new to the NPs. Priorities of the business were not always the priorities of the NPs, for example, using the video equipment was strongly encouraged by the organization because they were using it on trial from a major telecommunications provider in the country; however, the NPs and their clients were less enthusiastic.

\section{Limitations}

This pilot project has obvious limitations primarily as a result of business goals conflicting with conventional research processes. Internal bias and problems with generalizability are evident. For example, participant recruitment processes were based on assumptions about each group of invitees and their likelihood of enrolling in this service as a paying client which limits generalizability. Convenience sampling relying on current clients and those close to the business leaders also limits its generalizability to bigger systems like the public health care system where population demographics are more diverse. Furthermore, 
comparisons based on the age, income, or general health status of participants is biased because equal representation for each cohort was not obtained.

Participant responses in the evaluation process may be influenced by current connections and contact with the organization. A recruitment strategy that targets those who influence consumerism within Calgary's affluent community, may also create bias in their responses to evaluation. Participants recruited via snowball sampling, specifically those initial contacts originate amongst company staff, may also have created bias in client feedback.

The company chose not to separate member and non-member data in the evaluation, which was unfortunate as not all enrollees were members of this private care organization and some clearly would have never been able to afford this service beyond the pilot. On the other hand, the non-member input would have provided relevant data in terms of generalizability to the greater public. In the end, and despite the internal biases, the company knows who its clients tend to be, and the priority was to produce outcome analytics to inform future business planning. Limitations in the study are coherent from the company's goal: to expand services using NPs as mobile health care providers.

\section{Discussion}

This pilot met the evaluative nature of its goals: to gauge client satisfaction of a mobile NP primary care service and to assess the financial viability of providing such a service.

\section{House Calls}

This pilot confirms the trend in the United States that suggests that medical house calls can be successfully provided by NPs, and that clients were comfortable and satisfied with medical home visits provided by nurse practitioners. House calls are not a new intervention; in this case, the house call has been reimagined using NPs as the provider along with the addition of a few services, like full physicals at home, collection of laboratory specimens, and continued contact via multiple media.

\section{Telehealth}

Both the clients and the NPs in this pilot were comfortable using technology to enhance access to care. Ensuring privacy and confidentiality through the use of secure email, videoconferencing and phone services was an essential requirement from both professional and legal perspectives. Text-messaging and phone-based video calls (like Facetime ${ }^{\mathrm{TM}}$ and Snapchat ${ }^{\mathrm{TM}}$ ) are not secure, nor can they be easily copied into a chart for documentation of a patient encounter, and so can not be incorporated into the options for communication at this time. Some studies have demonstrated that patients tend to be most comfortable with email for communicating with health care personnel: the youngest, those who have more 
health literacy and more education, tend to prefer secure email (Huan et al. 2015, Jenssen et al. 2016). Technology continues to evolve to meet the needs of consumers, and secure methods available for patient-provider communication will likely expand to provide more options over time.

\section{Convenience versus Actual Need}

Most NP-client encounters occurred in regard to common health concerns often seen in regular physician offices thereby reinforcing the concept of convenience as the driver of service acceptability rather than exceptional health care requirements like immobility or frailty.

The affluent, dual-income couples in mid-life who have children at home, and who value convenience were the most willing to pay for this service. It is acknowledged that a lack of diversity amongst study participants in terms of age, income and health status skewed the results in a way that might have shown a greater need for the services based on health status if more of the participants had been elderly or unwell. However, the internal bias is consistent with the company's target cohort: the young, affluent and technologically savvy. The results confirm that their target customers are interested in this service and are willing to pay a fee for it.

\section{Bigger Picture Contributions}

It has been shown that accessible care in one's community reduces emergency department use for problems that could be taken care of in a regular primary care setting (Health Council of Canada 2014), this study could not conclude that accessible care provided by the NPs $24 / 7$ prevented use of the public health care system for routine or emergent care. In addition, the affluent typically have better health than the non-affluent, and the former are not known to frequent emergency facilities for routine care and are less likely to need emergent care due to deteriorating health (Woolf et al. 2015). The lack of hospitals and emergency room use for acute conditions was not surprising given the short duration of the pilot and the general good health of the study participants. No control group in the general public was provided for in this study for comparison in regard to hospitalization rates or emergency room use.

\section{Generalizability to the Public Health Care System}

Publicly-funded health care systems innovate to make improvements in population health while balancing the need to provide high-quality healthcare with cost-containment and maximum efficiency (Omachonuand and Einspruch 2010). In Canada, public healthcare services typically target the largest users of expensive resources: mainly the aged and people at the lower end of the socioeconomic ladder (CIHI 2014). So, while the busy, affluent client is sometimes willing to pay for concierge health care services like house calls, there may not be much advantage to targeting this demographic if the aim is reducing illnesses that result 
in hospital admission or emergency room care. Private health care organizations may continue to offer these enhanced primary care services to their clients, however, the not-for-profit public sector may find better value in offering house calls to specific populations like the housebound, the infirm, frail or elderly. More research is needed.

\section{Feasibility}

The service is practically and logistically feasible and is an effective means of providing high-quality health care services that is pleasing to clients. However, the service may not be economically feasible in the current model: i.e., an intake house call, that includes history-taking and a physical assessment, followed by health care services provided on demand either in-home or using various media. The cost of the delivering the current model of service, $\$ 120$ per hour, plus an added profit for the company, likely requires adjustment of the service model and options for pricing to make the business viable. The company can consider options such as an annual membership fee, or a fee-for-service model offering various "price packages" or a combination thereof, e.g., unlimited media contacts for a base price, with a separate fee for house calls.

\section{Conclusion}

Despite the flaws, this pilot suggests that medical house calls can be successfully provided by NPs in Canada and may be financially feasible within a user-pay system. There are limits on its generalizability of the findings, but this pilot may provide information useful to the public health care system if it chooses to utilize house calls as an adjunct to existing home care programs, or to augment care provided in physician clinics in both urban, rural and remote settings, and to expand the services available at Nurse Practitioner-Led Clinics in Ontario. It seems prudent to study this option further within the public sector embracing NPs and various secure media.

\section{Addendum}

The services described in this paper have since been reconfigured in a way that reduced costs, and focuses on video and phone consultations, with optional home or office visits for an additional fee. The service continues to utilize nurse practitioners and is currently being expanded across the country as a private health care service.

\section{Acknowledgements}

Our thanks to Dr. Ruth Martin-Misener at Dalhousie University, for permission to use the Misener NP Job Satisfaction Tool for this project. 
Vol. 6, No. 1 Clare: Bringing Health Care Home: A Pilot Project to Improve Access...

\section{References}

Bryant-Lukosius D, Dicenso A (2004) A framework for the introduction and evaluation of advanced practice nursing roles. Journal of Advanced Nursing 48(5): 530-540.

CHSRF-Canadian Health Services Research Foundation (2011) Better with age: health systems planning for the aging population. Synthesis report. Retrieved from https://bit.ly/2YaQ0Y1.

CIHI-Canadian Institute for Health Information (2014) Pan-Canadian forum on high users of health care: summary report. Retrieved from https://bit.ly/2TYWs51.

Canadian Nurses Association (2013) Optimizing the role of nursing in home health. Retrieved from https://bit.ly/2JsWeiy.

Chapman H, Farndon L, Matthews R, Stephenson J (2018) Okay to stay? A new plan to help people with long-term conditions remain in their own homes. Primary Health Care Research and Development 20: e16.

Desai NR, Smith KL, Boal J (2008) The positive financial contribution of home-based primary care programs: the case of the Mount Sinai Visiting Doctors. Journal of the American Geriatric Society 56(4): 744-749.

Government of Canada (2012) Innovation, Science and Economic Development Canada: Office of Consumer Affairs. Chapter 2 - Consumers and changing retail markets. Ottawa: Government of Canada.

Heale R, James S, Wenghofer E, Garceau M (2018) Nurse practitioner's perceptions of the impact of the nurse practitioner-led clinic model on the quality of care of complex patients. Primary Health Care Research and Development 19(6): 553-560.

Health Canada (2005) Unleashing innovation: excellent healthcare for Canada. Report of the advisory panel on healthcare innovation. Retrieved from https://bit.ly/2jX1YR0.

Health Council of Canada (2014) Where you live matters: Canadian views on health care quality, Bulletin 8 . Retrieved from https://bit.ly/2HHea6y.

Herritt B (2015) The house call: past, present and future. University of Toronto Medical Journal 89(3): 175-177.

Huan JN, Patel NR, Lind JD, Antinori N (2015) Large-scale survey findings inform patients' experiences in using secure messaging to engage in patient-provider communication and self-care management: a quantitative assessment. Journal of Medical Internet Research 17(12): e282.

Jenssen BP, Mitra N, Shah A, Wan F, Grande D (2016) Using digital technology to engage and communicate with patients: a survey of patient attitudes. Journal of General Internal Medicine 31(1): 85-92.

Leff B, Carlson C, Saliba D, Ritchie C (2015) The invisible homebound: setting qualityof-care standards for home-based primary and palliative care. Health Affairs 34(1): 21-29.

Lister C, Payne H, Hanson C, Barnes M, Davis S, Manwaring T (2017) The public health innovation model: merging private sector processes with public health strengths. Frontiers in Public Health 5(192): 1-9.

Martin-Misener R (2000) Misener nurse practitioner job satisfaction scale. (Used with permission). Retrieved from https://bit.ly/2OemrA8.

Meyers A (2015) 10 Reasons why house calls are a bad idea. MD Magazine [On-Line Article]. Retrieved from https://bit.ly/2TU86zf.

Musser S (2019) Prosperity coaching. [On-line Article]. Retrieved from https://bit.ly/ 2JrLwcf. 
Norman GJ, Orton K, Wade A, Morris AM, Slaboda JC (2018) Operation and challenges of home-based medical practices in the US: findings from six aggregated case studies. BMC Health Services Research 18(1): 45.

Omachonu V, Einspruch N (2010) Innovation in healthcare delivery systems: a conceptual framework. The Innovation Journal: The Public Sector Innovation Journal 15(2): 120.

Sandomirsky D (2018) Top ten healthcare game changers - Canada's emerging health innovations and trends. Hospital and Healthcare Management. Retrieved from https://bit.ly/2FiymsG.

Schuchman M, Fain M, Cornwell T (2018) The resurgence of home-based primary care in the United States. Geriatrics 3(41): 1-10.

Smith C, Dhalla I (2011) Why don't more doctors do house calls? Health Debate [OnLine Article]. Retrieved from https://bit.ly/2OjsGCJ.

Stall N, Nowaczynski M, Sinha S (2013) Commentary - Back to the future: Home-based primary care for older homebound Canadians. Canadian Family Physician 59(3): 237-240.

Walker B (2018) Insights on today's healthcare consumer: How to engage millennial healthcare consumers in 2018. Retrieved from https://bit.ly/2ugFscf.

Woolf SH, Aron L, Dubay L, Simon SM, Zimmerman E, Luk K (2015) How income and wealth are linked to health and longevity. Income and Health Initiative: Brief One. New York: Virginia Commonwealth University.

Yao NA, Rose K, LeBron V, Camacho F, Boling P (2016) Increasing role of nurse practitioners in house call programs. Journal of the American Geriatric Society 65(4): 847-852. 
